\title{
Long-term outcomes of emergency ABO-incompatible living donor liver transplantation using a modified desensitization protocol for highly sensitized patients with acute liver failure: A case report
}

\author{
Boram Lee', Jai Young Cho', Ho-Seong Han', Yoo-Seok Yoon', Hae Won Lee', Jun Suh Lee', Moonhwan Kim', YoungRok Choi² \\ 'Department of Surgery, Seoul National University Bundang Hospital, Seoul National University College of Medicine, Seongnam, Korea, \\ ${ }^{2}$ Department of Surgery, Seoul National University Hospital, Seoul National University College of Medicine, Seoul, Korea
}

\begin{abstract}
Although there is no established desensitization protocol for liver transplantation (LT), desensitization usually entails treatment with rituximab, plasmapheresis, splenectomy, and intravenous immunoglobulin (IVIG) infusion together with a local graft. The desensitization protocol is usually initiated 2 to 3 weeks before transplantation. Therefore, patients with acute liver failure warranting urgent LT are usually ineligible for ABO-incompatible (ABOi) LT. For these reasons, several attempts have been made to abridge the desensitization protocol and extend the indication for ABOi living donor LT (LDLT). Here we report a 40-year-old female diagnosed with chronic hepatitis B and acute-on-chronic liver failure (model for end-stage liver disease score, 31). In the absence of a suitable compatible liver donor, emergency ABOi LT was planned using a modified desensitization protocol. The preoperative isoagglutinin (IA) titer was $1: 1,024$ and the preoperative T- and B-cell cross-matches were positive. The patient received a single dose of rituximab (375 $\mathrm{mg} /$ $\left.\mathrm{m}^{2}\right)$ and IVIG $(0.8 \mathrm{~g} / \mathrm{kg})$ was administered from the anhepatic phase until three days after transplantation. Although the patient developed acute cellular rejection in the early stages after LT, she has maintained a stable graft function, even after 5 years. In summary, a modified desensitization protocol consisting of rituximab and IVIG is a feasible strategy for highly sensitized patients with elevated IA titers indicated for urgent LDLT.
\end{abstract}

Key Words: Liver transplantation; Graft rejection; Immunoglobulins, intravenous; Liver failure, acute

\section{INTRODUCTION}

Since the first-reported ABO-incompatible (ABOi) liver transplantation (LT) by Gordon et al. [1] in the 1980s, liver has been regarded as an immunologically privileged organ. However, the high incidence of early graft loss due to antibody-me-

Received: December 24, 2020, Revised: July 21, 2021

Accepted: July 26, 2021

Corresponding author: Jai Young Cho

Department of Surgery, Seoul National University Bundang Hospital,

82 Gumi-ro 173beon-gil, Bundang-gu, Seongnam 13620, Korea

Tel: +82-31-787-7098, Fax: +82-31-787-4078, E-mail: jychogs@gmail.com

ORCID: https://orcid.org/0000-0002-1376-956X

Copyright (C) The Korean Association of Hepato-Biliary-Pancreatic Surgery diated rejection (AMR) was a major concern in ABOi LT [2]. Since the introduction of rituximab (an anti-CD20 monoclonal antibody) in the 2000s, the incidence of AMR has decreased dramatically and the indications for ABOi LT have increased [2]. ABOi LT has been used routinely in recent years with acceptable outcomes to overcome the limited organ availability.

In the absence of an established desensitization protocol, treatment usually entails administration of rituximab, plasmapheresis, splenectomy, and intravenous immunoglobulin (IVIG) [3]. Because the desensitization protocol is usually started 2 to 3 weeks before transplantation, ABOi LT is considered impossible in patients with acute liver failure (ALF). For these reasons, several centers have attempted modified desensitization protocols. Shen et al. [4] reported that a protocol comprising a single dose of rituximab and IVIG at the start of LT, followed by ongoing IVIG for 10 consecutive days was effective in patients with ALF. Kim et al. [5] reported successful 
outcomes with a modified protocol using rituximab and IVIG. These protocols omitted plasmapheresis before transplantation, and showed sufficient desensitization for ABOi LT using modified protocols. However, most of the patients included in those studies had low initial isoagglutinin (IA) titers. Furthermore, few reports have described the long-term outcomes of ABOi LT based on a modified desensitization protocol.

In this case report, we describe a highly sensitized patient with elevated IA titers who underwent ABOi LT using a modified desensitization protocol for ALF. We also report the longterm outcomes in this patient.

\section{CASE}

A 40-year-old female (blood type, $\mathrm{Rh} \mathrm{O}+$ ) undergoing treatment for chronic hepatitis B presented at our emergency department with a 1-week history of abdominal pain. On admission, her total bilirubin (T-bil) was $4.4 \mathrm{mg} / \mathrm{dL}$, and her international normalized ratio was 2.17 . The model for end-stage liver disease (MELD) score was 31. She developed spontaneous bacterial peritonitis during admission. Despite treatment, she progressed to type 1 hepatorenal syndrome with grade 1 hepatic encephalopathy and a MELD score of 35 . Following a multidisciplinary team discussion, we planned to perform emergency living donor LT (LDLT). However, in the absence of suitable compatible liver donors, her 39-year-old husband with blood type A+ was used as the living donor. Patient consent for the use of retrospective hospital data was not necessary for this study.

The patient's initial immunoglobulin G (IgG) and immunoglobulin $\mathrm{M}(\operatorname{IgM})$ titers were $1: 1,024$ and $1: 512$, respectively. The IA titer was measured by column agglutination method. The preoperative T- and B-cell cross-matches were positive. The panel reactive antibodies (PRA) were $100 \%$ for classes I and II. Unfortunately, we could not validate the donor-specific antibody (DSA) results before transplantation. The percentage of cluster of differentiation 19 (CD19) was 14 before administering rituximab. The preoperative examination confirmed that she was a sensitized patient. However, we decided to proceed with a modified desensitization protocol, because her condition worsened rapidly (Fig. 1). Rituximab $\left(375 \mathrm{mg} / \mathrm{m}^{2}\right)$ was administered 3 days before LT and IVIG $(0.8 \mathrm{~g} / \mathrm{kg})$ was infused from the day of LT in the anhepatic phase until 3 days after transplantation. Although her baseline IA titer was high, plasmapheresis was omitted. The emergency ABOi LDLT was completed without complications. The estimated blood loss was $1,700 \mathrm{~mL}$. The patient received about 6 pints of packed red blood cells, 6 pints of fresh frozen plasma, and 1 pint of apheresis platelets. After transplantation, the percentage of CD19 was 1 and the IgG/IgM titers were $1: 64$ and $1: 16$ on postoperative day (POD) 7. Post-transplant immunosuppression was achieved using tacrolimus, corticosteroid and mycophenolate

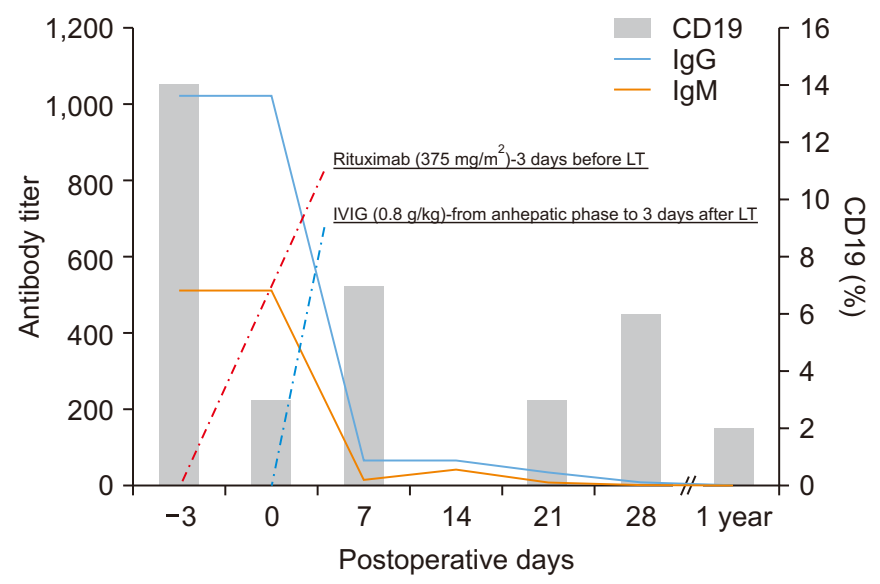

Fig. 1. Modified perioperative $A B O$-incompatible protocol and perioperative immunoglobulin and CD19 profile. CD19, cluster of differentiation 19; IVIG, intravenous immunoglobulin; IgG, immunoglobulin $\mathrm{G}$; IgM, immunoglobulin $\mathrm{M}$; LT, liver transplantation.

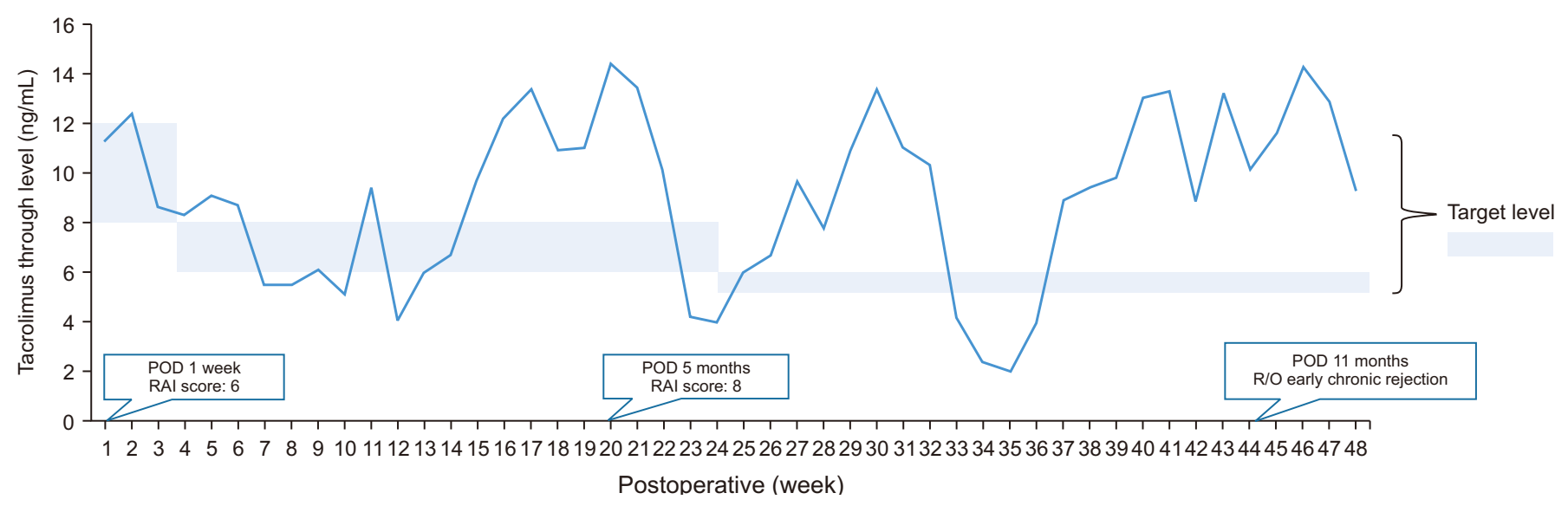

Fig. 2. Serum tacrolimus level within 1 year after liver transplantation. POD, postoperative day; RAI, rejection activity score; R/O, rule out. 
mofetil (Fig. 2). The dose of tacrolimus was usually adjusted to maintain a level of 8 to $12 \mathrm{ng} / \mathrm{mL}$ during the first month after transplantation, and 6 to $8 \mathrm{ng} / \mathrm{mL}$ during the months 1 to 6 after transplantation in our institution. Since the patient was at high risk of rejection, the dose of tacrolimus was adjusted higher than in other patients. However, the protocol biopsy on POD 7 showed acute cellular rejection (ACR) (rejection activity score, 6) with elevated hepatic enzymes (T-bil, $10.0 \mathrm{mg} / \mathrm{dL}$; aspartate aminotransferase [AST], 65 IU/L; alanine aminotransferase [ALT], $111 \mathrm{IU} / \mathrm{L})$. She received steroid pulse therapy (500 mg hydrocortisone for 3 days, followed by tapering). The follow-up biopsy on POD 14 showed improved ACR and hepatic enzyme levels. She was discharged on POD 22, at which time her IgG and IgM titers were $1: 32$ and $1: 8$, respectively.

Five months after transplantation, the patient's graft function worsened. A follow-up biopsy showed ACR (rejection activity score, 8) with mild elevation of hepatic enzyme (T-bil, $2.3 \mathrm{mg} /$ dL; AST, 440 IU/L; ALT, 500 IU/L). The titers of IgG and IgM remained stable (1:8 and $1: 2$, respectively). She received steroid pulse therapy (100 mg hydrocortisone for 3 days, followed by tapering). The treatment for ACR was followed by discharge due to decreased hepatic enzymes. At that time, the patient converted to negative crossmatch and negative DSA during a 6-months post-transplant test.

Eleven months after transplantation, she was hospitalized due to abnormal hepatic enzymes (T-bil, $3.2 \mathrm{mg} / \mathrm{dL}$; alkaline phosphatase [ALP], 376 IU/L; AST, 96 IU/L; ALT, 108 IU/L; $\gamma$-glutamyltransferase $[\gamma \mathrm{GT}], 607 \mathrm{IU} / \mathrm{L})$ and underwent liver biopsy. The pathologic reports suggested the possibility of early chronic rejection. At this time, her IgG and IgM titers were 1 : 4 and $1: 1$, respectively. Although, the histopathological examination revealed minimal ( $<10 \%$ portal tracts) $\mathrm{C} 4 \mathrm{~d}$ deposit, no human leukocyte antigen (HLA) DSA was detected. Therefore, the possibility of AMR was ruled out. Even though chronic rejection was suspected, the patient manifested no abnormal signs other than elevated hepatic enzymes, and was therefore treated with an increasing dose of immunosuppressive agents without steroid pulse therapy. Five days after treatment, her hepatic enzyme levels had decreased (T-bil, $1.4 \mathrm{mg} / \mathrm{dL}$; ALP, 302 IU/L; AST, 62 IU/L; ALT, 89 IU/L; $\gamma \mathrm{GT}, 500 \mathrm{IU} / \mathrm{L})$.

A follow-up liver biopsy 1.5 years after transplantation revealed no evidence of graft rejection. Five years after LDLT, the patient and the graft were stable.

\section{DISCUSSION}

The desensitization protocol for ABOi LDLT usually takes 2 to 3 weeks. Thus, patients with ALF warranting urgent LT are generally considered ineligible for ABOi LDLT [6]. Therefore, several attempts have been made to shorten the desensitization period and extend the indications for ABOi LDLT in ALF. The desensitization period is mainly attributed to plasmapheresis, which reduces the IA titer before transplantation. It is thought that elevated IA titer before transplantation increases the risk of AMR [7,8], and IA titers below $1: 16$ or $1: 32$ are considered safe. However, recent studies reported no significant correlation between the IA titer and AMR $[9,10]$. Consequently, several centers have proposed modified desensitization protocols [11-13]. The omission of plasmapheresis is a prominent change in these protocols. Indeed, modified protocols based on rituximab monotherapy resulted in sufficient desensitization to enable ABOi LDLT. However, the mean IA titers prior to LT were relatively low (less than $1: 64$ and $1: 96$ ). In addition, the MELD scores of patients in these studies were 10-16. Therefore, it is unclear whether rituximab monotherapy is sufficient for patients with high MELD scores and elevated IA titers requiring urgent LT.

Shen et al. introduced a modified desensitization protocol consisting of rituximab and IVIG for emergent ABOi LT in patients with ALF [4]. The mean MELD score and IA titers were 34.3 and $1: 192$, respectively. In contrast to previous studies, Shen et al. reported favorable outcomes in patients with relatively high IA titers before urgent LT. They administered IVIG $(0.4 \mathrm{~g} / \mathrm{kg}$ per day) from the start of LT as induction therapy to reduce the IA titer. IVIG may induce direct effects on circulating immunoglobulin and attenuate immunomodulatory mechanisms by reducing helper CD4 $\mathrm{T}$ cell activity or suppress $\mathrm{T}$ cell alloreactivity by inhibiting cytokine production [14]. IVIG may also play a key role in reducing the IA titer after transplantation if plasmapheresis is not available. Therefore, in this case, the IA titer was maintained at a low level for more than 1 year after transplantation, despite the increasing of B cells.

Our patient had an extremely high IA titer and was crossmatch-positive before transplantation, unlike those reported in previous studies. In addition, plasmapheresis was omitted due to the risk of wash-out effect [15]. Therefore, we used a higher dose of IVIG ( $0.8 \mathrm{~g} / \mathrm{kg}$ per day) to prevent hyperacute or acute rejection and subsequent graft loss. Until now, few studies reported the optimal dose and timing of IVIG administration. An appropriate dosage of IVIG was needed to prevent infection and AMR. Although it is unclear whether a dose of $0.8 \mathrm{~g} / \mathrm{kg}$ per day was appropriate, our patient did not experience a rebound increase in antibody titers after transplantation, and her IA titer remained low.

In general, sustained B-cell depletion is observed up to 3-9 months post-transplantation in $100 \%$ of patients. B cell recovery occurs starting at approximately $5.3 \pm 2.9$ months following completion of treatment and median B-cell levels return to normal by a mean time of $\sim 12$ months following completion of treatment [16]. However, in this case, the CD 19 count was not suppressed effectively. Actually, the mechanism of resistance to rituximab in vivo is not clear. Several mechanisms have been reported including inhibition of antibody-dependent cellular cytotoxicity via deposition of C3 activating fragments, FcrRIIIa polymorphism in cytotoxic cells, inhibition of CDC, and loss of CD 20 expression on the surface of subclones [17-19]. Some 
of the mechanisms of resistance to rituximab may or may not be applicable in vivo, and therefore need to be validated.

In conclusion, we have successfully performed emergency ABOi LDLT in a highly sensitized patient with a high IA titer using a modified desensitization protocol. Although the patient experienced ACR, her long-term outcomes, evaluated at 60 months after LT, were optimal and her graft function was normal. Thus, a modified desensitization protocol consisting of rituximab and IVIG is a feasible strategy for highly sensitized patients with a high IA titer warranting urgent LT.

\section{CONFLICT OF INTEREST}

No potential conflict of interest relevant to this article was reported.

\section{ORCID}

Boram Lee, https://orcid.org/0000-0003-1567-1774

Jai Young Cho, https://orcid.org/0000-0002-1376-956X

Ho-Seong Han, https://orcid.org/0000-0001-9659-1260

Yoo-Seok Yoon, https://orcid.org/0000-0001-7621-8557

Hae Won Lee, https://orcid.org/0000-0002-3312-9295

Jun Suh Lee, https://orcid.org/0000-0001-9487-9826

Moonhwan Kim, https://orcid.org/0000-0003-2772-0836

YoungRok Choi, https://orcid.org/0000-0003-2408-7086

\section{AUTHOR CONTRIBUTIONS}

Conceptualization: All authors. Data curation: All authors. Methodology: All authors. Visualization: BL, JYC. Writing original draft: All authors. Writing - review \& editing: JYC, BL.

\section{REFERENCES}

1. Gordon RD, Iwatsuki S, Esquivel CO, Tzakis A, Todo S, Starzl TE. Liver transplantation across ABO blood groups. Surgery 1986;100:342-348.

2. Egawa H, Teramukai S, Haga H, Tanabe M, Mori A, Ikegami T, et al. Impact of rituximab desensitization on blood-type-incompatible adult living donor liver transplantation: a Japanese multicenter study. Am J Transplant 2014;14:102-114.

3. Usuda M, Fujimori K, Koyamada N, Fukumori T, Sekiguchi S, Kawagishi N, et al. Successful use of anti-CD20 monoclonal antibody (rituximab) for ABO-incompatible living-related liver transplantation. Transplantation 2005;79:12-16.

4. Shen T, Lin BY, Jia JJ, Wang ZY, Wang L, Ling Q, et al. A modified protocol with rituximab and intravenous immunoglobulin in emergent $\mathrm{ABO}$-incompatible liver transplantation for acute liver failure. Hepatobiliary Pancreat Dis Int 2014;13:395-401.

5. Kim SH, Lee EC, Shim JR, Park SJ. A simplified protocol using rituximab and immunoglobulin for ABO-incompatible low-titre living donor liver transplantation. Liver Int 2018;38:932-939.
6. Song GW, Lee SG, Hwang S, Kim KH, Ahn CS, Moon DB, et al. ABO-incompatible adult living donor liver transplantation under the desensitization protocol with rituximab. Am J Transplant 2016;16:157-170.

7. Egawa H, Teramukai S, Haga H, Tanabe M, Fukushima M, Shimazu M. Present status of ABO-incompatible living donor liver transplantation in Japan. Hepatology 2008;47:143-152.

8. Haga H, Egawa H, Fujimoto Y, Ueda M, Miyagawa-Hayashino A, Sakurai T, et al. Acute humoral rejection and C4d immunostaining in ABO blood type-incompatible liver transplantation. Liver Transpl 2006;12:457-464.

9. Skogsberg U, Breimer ME, Friman S, Mjörnstedt L, Mölne J, Olausson $\mathrm{M}$, et al. Adult $\mathrm{ABO}$-incompatible liver transplantation, using $\mathrm{A}$ and B donors. Xenotransplantation 2006;13:154-159.

10. Mendes M, Ferreira AC, Ferreira A, Remédio F, Aires I, Cordeiro A, et al. ABO-incompatible liver transplantation in acute liver failure: a single Portuguese center study. Transplant Proc 2013;45:1110-1115.

11. Yamamoto H, Uchida K, Kawabata S, Isono K, Miura K, Hayashida $\mathrm{S}$, et al. Feasibility of monotherapy by rituximab without additional desensitization in ABO-incompatible living-donor liver transplantation. Transplantation 2018;102:97-104.

12. Lee EC, Kim SH, Shim JR, Park SJ. A comparison of desensitization methods: rituximab with/without plasmapheresis in ABO-incompatible living donor liver transplantation. Hepatobiliary Pancreat Dis Int 2018;17:119-125.

13. Lee B, Choi Y, Han HS, Yoon YS, Cho JY, Jeong SH, et al. ABO-incompatible liver transplantation using only rituximab for patients with low anti-ABO antibody titer. Ann Hepatobiliary Pancreat Surg 2019;23:211-218.

14. John R, Lietz K, Burke E, Ankersmit J, Mancini D, Suciu-Foca N, et al. Intravenous immunoglobulin reduces anti-HLA alloreactivity and shortens waiting time to cardiac transplantation in highly sensitized left ventricular assist device recipients. Circulation 1999;100(19 Suppl):II229-II235.

15. Puisset F, White-Koning M, Kamar N, Huart A, Haberer F, Blasco $\mathrm{H}$, et al. Population pharmacokinetics of rituximab with or without plasmapheresis in kidney patients with antibody-mediated disease. Br J Clin Pharmacol 2013;76:734-740.

16. Zarkhin V, Li L, Kambham N, Sigdel T, Salvatierra O, Sarwal MM. A randomized, prospective trial of rituximab for acute rejection in pediatric renal transplantation. Am J Transplant 2008;8:2607-2617.

17. Wang M, Han XH, Zhang L, Yang J, Qian JF, Shi YK, et al. Bortezomib is synergistic with rituximab and cyclophosphamide in inducing apoptosis of mantle cell lymphoma cells in vitro and in vivo. Leukemia 2008;22:179-185.

18. Cartron G, Dacheux L, Salles G, Solal-Celigny P, Bardos P, Colombat $\mathrm{P}$, et al. Therapeutic activity of humanized anti-CD20 monoclonal antibody and polymorphism in IgG Fc receptor FcgammaRIIIa gene. Blood 2002;99:754-758.

19. Weng WK, Levy R. Two immunoglobulin G fragment C receptor polymorphisms independently predict response to rituximab in patients with follicular lymphoma. J Clin Oncol 2003;21:3940-3947. 